\title{
Compact sets with vanishing cohomology in Stein spaces and domains of holomorphy
}

\author{
VIOREL VÂJÂITU
}

\begin{abstract}
Let $X$ be a Stein space. We study compact subsets $K$ of $X$ that are structurally acyclic, i.e., $H^{i}\left(K, \mathcal{O}_{X}\right)=0$, for all $i \geq 1$. We show i) that such compact sets are natural in the sense that the canonical map from $K$ into $\widetilde{K}$, the spectrum of the complex algebra $\Gamma\left(K, \mathcal{O}_{X}\right)$, is bijective, and ii) that the set of interior points of $K$ is a domain of holomorphy in $X$. Motivated by this we give an extensive account of examples of domains of holomorphy in non-normal Stein spaces and prove several properties, like hereditarity via the normalization map. Finally, a straightforward criterion of non-acyclicity is given in terms of general Hartogs figures.
\end{abstract}

Mathematics Subject Classification (2010): 32D05 (primary); 32C35, 32E10, 32C15 (secondary).

\section{Introduction}

Let $X$ be a Stein manifold of pure dimension $n \geq 2$. Let $\Omega$ be a relatively compact (strongly) pseudoconvex open subset of $X$ with smooth boundary $\partial \Omega$ of class $C^{\infty}$. One important theme in Complex Analysis is the characterization of compact subsets $K$ of $\bar{\Omega}$ with $\partial \Omega \not \subset K$ such that one has holomorphic continuation of CR-functions, or more generally forms, from $\partial \Omega \backslash K$ to $\Omega \backslash K$, see [25-27].

For instance in [25] the solvalility of the tangential Cauchy-Riemann in every bidegree is considered assuming that $\Omega \backslash K$ is Stein and $n \geq 3$ (see conditions (A1), (A2) and (A3) on page 1064; e.g., (A1) means extension of continuous CRfunctions on $\partial \Omega \backslash K$ to continuous functions on $\bar{\Omega} \backslash K$ that are holomorphic on $\Omega \backslash K)$. This is given in terms of the vanishing of Dolbeault cohomology groups $H^{n, q}(K)$, for $1 \leq q<n$, so that a substantial portion of that article explores the vanishing condition. More precisely the following result is given [25, pages 10731077]:

Received March 30, 2011; accepted in revised form November 21, 2011. 
Theorem. Let $X$ be a connected Stein manifold of dimension n. Let $K \subset X$ be a compact set satisfying the following three conditions:

a) $K$ is structurally acyclic;

b) $K$ is fat, in the sense that $K$ equals the closure of its interior, and each point $x_{0} \in \partial K$ has a system of open neighborhoods having connected intersection with the interior of $K$;

c) the map $\pi$ from $\widetilde{K}$ to $X$ is locally a homeomorphism and $\widetilde{K}$ coincides with the closure of the pull-back of the interior of $\pi(\widetilde{K})$.

Then $K$ has a nonschlicht Stein neighborhood basis.

Note. For the commodity of the reader we recall the following. Let $K$ be a compact set in a Stein manifold $X$.

- We say that $K$ has a nonschlicht Stein neighborhood basis if for every open neighborhood $U$ of $K$ in $X$ there exists another open neighborhood $W$ of $K$ in $U$ such that $\pi(\widetilde{W})$ is contained in $U$, where $\pi: \widetilde{W} \rightarrow X$ is the envelope of holomorphy of $W$.

- $\widetilde{K}$ denotes the spectrum of $\Gamma\left(K, \mathcal{O}_{X}\right)$, that is the set of all non-zero multiplicative linear functionals on $\Gamma\left(K, \mathcal{O}_{X}\right)$.

Remark 1.1. In order to relate the vanishing of the Dolbeault cohomology groups and those with coefficients in the structural sheaf we note that if $\mathcal{L}$ is an invertible sheaf on $X$, then for a compact set $K$ in $X$ and a positive integer $j$, the cohomology groups $H^{j}(K, \mathcal{O})$ and $H^{j}(K, \mathcal{L})$ vanish simultaneously or not. The proof readily follows from the following facts:

First, if $X$ is a Stein space of finite dimension and $\mathcal{F}^{\prime \prime}$ is a locally free analytic sheaf of finite rank, then there is another locally free analytic sheaf $\mathcal{F}^{\prime}$ of finite rank such that $\mathcal{F}^{\prime} \oplus \mathcal{F}^{\prime \prime}$ is globally free. For this, observe that there are finitely many global holomorphic sections $s_{1}, \ldots, s_{r}$ of $\mathcal{F}^{\prime \prime}$ whose germs at an arbitrary point $x \in X$ generate the fiber $\mathcal{F}_{x}^{\prime \prime}$. It results, then, that the kernel $\mathcal{F}^{\prime}$ of the canonical $\mathcal{O}_{X}$-morphism from $\mathcal{O}_{X}^{r}$ onto $\mathcal{F}^{\prime \prime}$ is locally free. By the Theorem B of Cartan and Serre, the short exact sequence

$$
0 \rightarrow \Gamma\left(X, \mathcal{F}^{\prime}\right) \rightarrow \Gamma\left(X, \mathcal{O}_{X}^{r}\right) \rightarrow \Gamma\left(X, \mathcal{F}^{\prime \prime}\right) \rightarrow 0
$$

splits, so that we get a non-canonical isomomorphism $\mathcal{F}^{\prime} \oplus \mathcal{F}^{\prime \prime} \simeq \mathcal{O}_{X}^{r}$.

Secondly, if $X$ is non-singular and connected and $\mathcal{F}^{\prime \prime}$ an invertible sheaf on $X$, like the sheaf of germs holomorphic sections of its cotangent bundle, from $\mathcal{O}^{r} \simeq$ $\mathcal{F}^{\prime} \oplus \mathcal{F}^{\prime \prime}$ and $\operatorname{Hom}_{\mathcal{O}}\left(\mathcal{F}^{\prime \prime}, \mathcal{F}^{\prime \prime}\right) \simeq \mathcal{O}$, we obtain $\left(\mathcal{F}^{\prime \prime}\right)^{r} \simeq \operatorname{Hom}_{\mathcal{O}}\left(\mathcal{F}^{\prime}, \mathcal{F}^{\prime \prime}\right) \oplus \mathcal{O}$.

One goal of this paper is to show that the very technical conditions b) and c) in the above theorem are superfluous. Moreover we shall extend and partially answer a question in [25], in the more general setting where the ambient space admits singularities. More precisely we prove (for notation and definitions see Section 2): 
Theorem 1.2. Let $X$ be a holomorphically spreadable complex space and $K \subset X$ a compact set. Then $K$ is structurally acyclic if and only if $K$ is natural with respect to the reduced complex structure of $X$. Moreover, if $X$ is Stein and nonsingular, then each of these conditions is equivalent to saying that $K$ has a nonschlicht Stein neighborhood basis.

Theorem 1.3. Let $K$ be a natural compact set in a Stein space $X$ of pure dimension. Then $\operatorname{int}(K)$, the interior of $K$, is a domain of holomorphy in $X$. In particular, if $X$ is non-singular, then $\operatorname{int}(K)$ is Stein.

Theorem 1.2 answers a question raised in [25, page 1077] and Theorem 1.3 improves Théorème 2.6 in the same paper.

\section{Preliminaries}

Throughout this paper complex spaces, unless otherwise specified, are non-necessarily reduced. A curve, surface, etc., will be a complex space of the appropriate pure dimension.

Following [29] we consider Rs to be the full subcategory of the category of ringed spaces in local $\mathbb{C}$-algebras having as objects the restriction subspaces of complex spaces; thus a ringed space $\left(S, \mathcal{O}_{S}\right)$ is an object of $\mathbf{R s}$ if there is a complex space $\left(X, \mathcal{O}_{X}\right)$ such that $S$ is a topological subspace of $X$ and $\mathcal{O}_{S}$ is the topological sheaf restriction of $\mathcal{O}_{X}$ to $S$, customarily denoted by $\left.\mathcal{O}_{X}\right|_{S}$. Notice that $X$ is individualized only as a germ about $S$.

In most cases encountered throughout this paper, $S$ will be closed in some complex representative; a fortiori this is fulfilled when $S$ is compact.

If $\varphi: S \rightarrow T$ is a morphism in Rs, then $\varphi$ is induced by a holomorphic map $f: X \rightarrow Y$ for suitable complex spaces $X$ and $Y$ along $S$ and $T$ that define $S$ and $T$ respectively. (See [29, Proposition 2.3].)

Let $S=\left(S, \mathcal{O}_{S}\right)$ be a space in Rs and $X=\left(X, \mathcal{O}_{X}\right)$ be a complex space representative of $S$. On the same underlying topological space $X$ we may have several complex structures, each of which induces a corresponding one on $S$. For instance to $\left(X, \mathcal{O}_{X}\right)$ it corresponds the reduction $\overline{\mathcal{O}}_{X}:=\mathcal{O}_{X_{\text {red }}}=\mathcal{O}_{X} / \mathcal{N}$, where $\mathcal{N}$ is the ideal subsheaf of $\mathcal{O}_{X}$ of germs of nilpotents elements; hence we obtain $\left(S, \overline{\mathcal{O}}_{S}\right)$.

More generally, we say that a coherent analytic sheaf $\mathcal{A}_{X}$ on $X$ is an infinitesimal extension of $\mathcal{O}_{X}$ if $\left(X, \mathcal{A}_{X}\right)$ is a complex space so that $\left(X, \mathcal{O}_{X}\right)$ becomes a closed subspace of $\left(X, \mathcal{A}_{X}\right)$. This means that there is a coherent sheaf of ideals $\mathcal{I} \subset \mathcal{A}_{X}$ such that $\operatorname{Supp} \mathcal{A}_{X} / \mathcal{I}=X$ and $\mathcal{O}_{X} \simeq \mathcal{A}_{X} / \mathcal{I}$ (ringed sheaves isomorphism). The support condition is equivalent by Rückert's Nullstellensatz to saying that $\mathcal{I} \subset \mathcal{N}$, where $\mathcal{N}$ is the nilradical of $\mathcal{A}_{X}$. For instance $\left(X, \mathcal{O}_{X}\right)$ is an infinitesimal extension of its reduction $\left(X, \overline{\mathcal{O}}_{X}\right)$.

We shall say that $\left(S, \mathcal{O}_{S}\right)$ is acyclic if $H^{j}(S, \mathcal{F})$ vanishes for all positive integers $j$ and all coherent sheaves $\mathcal{F}$ on $\left(S, \mathcal{O}_{S}\right)$. If we restrict the vanishing to $\mathcal{F}=\left.\mathcal{O}_{X}\right|_{S}$, then we call $S$ structurally acyclic. 
It is perhaps of importance to notice that if $S$ is closed in $X, e . g . S$ is compact, then a theorem due to Cartan [8] asserts that a ringed sheaf $\mathcal{F}$ of $\mathcal{O}_{S}$-modules on $S$ is coherent if and only if there exists an open neighborhood $U$ of $S$ in $X$ and a coherent sheaf $\widehat{\mathcal{F}}$ of $\mathcal{O}_{U}$-modules over $U$ such that $\left.\widehat{\mathcal{F}}\right|_{S}=\mathcal{F}$.

Now, to $\left(S, \mathcal{O}_{S}\right)$ in $\mathbf{R s}$, we associate a topological space $\tilde{S}$ of characters of the complex algebra of global sections $\Gamma\left(S, \mathcal{O}_{S}\right)$ i.e. all $\mathbb{C}$-algebra homomorphisms from $\Gamma\left(S, \mathcal{O}_{S}\right)$ into $\mathbb{C}$ (non-necessarily continuous) endowed with the coarsest topology such that, for each $s \in S$, the mapping

$$
\widehat{s}: \Gamma\left(S, \mathcal{O}_{S}\right) \rightarrow \mathbb{C}, \mathbf{h} \mapsto \widehat{s}(\mathbf{h}):=\mathbf{h}(s)
$$

is continuous. We therefore get a canonical continuous map

$$
\xi_{S}: S \rightarrow \widetilde{S}, s \mapsto \chi_{s}
$$

where $\chi_{s}$ is the evaluation character at $s$. (Here is the customary abuse of notation: The elements of the algebra $\Gamma\left(S, \mathcal{O}_{X}\right)$ are germs, i.e. equivalence classes of functions, not functions, so, strictly speaking $\mathbf{h}(s)$ is without meaning. Nonetheless, as usual, we understand by this symbol the value $h(s)$ for any function in the equivalence class $\mathbf{h}$. In various places $\widetilde{S}$ is also referred to as the envelope of holomorphy of $S$; this terminology is justified, for instance in [16] and [18]; mostly it is used for $S$ an open subset of $X$; it always exist if $X$ is non-singular and Stein; see also the last part of this section.)

Definition 2.1. Let $\left(K, \mathcal{O}_{K}\right)$ be a compact space in $\mathbf{R s}$ and $\left(X, \mathcal{O}_{X}\right)$, be a complex representative. We say that $K$ is natural in $X$ if:

- the canonical map $\xi_{K}: K \rightarrow \widetilde{K}$ is a homeomorphism and

- there exists an open neighborhood $U$ of $K$ in $X$ such that $\Gamma\left(U, \mathcal{O}_{X}\right)$ separates points in $U$.

The term "natural" is coined in [36]; but in use there are several labels like "holomorphically convex" (see $[18,30,41]$ ) or "holomorphic set" [7]. From the philosophical point of view, being natural for a compact space is a cohomological condition while ordinary holomorphic convexity is geometric. This hints for stability of naturality under two standard operations in complex analysis like reduction and normalization while for holomorphic convexity they fail!

The theory of natural sets has beed discussed, for instance, in the papers [18] and [41]. Examples in $\mathbb{C}^{n}$ include the polynomially convex sets, the rationally convex sets, and compact sets that are intersection of sequences of domains of holomorphy. The latter examples include all compact, totally real subsets of $\mathbb{C}^{n}$. Natural sets share the most important properties of Stein spaces, in that analogues of Cartan's Theorems A and B are valid for them. Here we summarize a part of the results from [29] (see also [41] for the reduced ambient setting). 
Theorem 2.2. Let $\left(K, \mathcal{O}_{K}\right)$ be a compact space in $\mathbf{R s}$ and $\left(X, \mathcal{O}_{X}\right)$ be a complex representative. Then the following assertions hold true:

$\alpha)$ If $\left(K, \mathcal{O}_{K}\right)$ is natural, then for every coherent sheaf $\mathcal{F}$ of $\mathcal{O}_{K}$-modules and every integer $i \geq 1$, the cohomology group $H^{i}(K, \mathcal{F})$ vanishes;

$\beta)$ The spaces $\left(K, \mathcal{O}_{K}\right)$ and $\left(K, \overline{\mathcal{O}}_{K}\right)$ are either both natural or both not;

$\gamma)$ If $H^{1}(K, \mathcal{F})$ vanishes for all coherent subsheaves $\mathcal{F}$ of $\mathcal{O}_{K}^{v}$, with $v \geq 1$, then $\left(K, \mathcal{O}_{K}\right)$ is natural.

The statement $\beta$ ) of above can be improved mutatis mutandis as in [29] to:

Proposition 2.3. Let $\left(X, \mathcal{A}_{X}\right)$ be an infinitesimal extension of $\left(X, \mathcal{O}_{X}\right)$. Let $K \subset$ $X$ be a compact set. Then $\left(K,\left.\mathcal{O}_{X}\right|_{K}\right)$ and $\left(K,\left.\mathcal{A}_{X}\right|_{K}\right)$ are either both natural on both not.

A classical theorem [22, page 267]; [16, pages 182-183]; [21] states that a reduced complex space $X$ of finite dimension is Stein if and only if the canonical map

$$
\xi_{X}: X \rightarrow \tilde{X}
$$

is a homeomorphism.

Thus for Stein $X$ we identify $X$ and $\widetilde{X}$ via the map $\xi_{X}$. Therefore, when $X$ is Stein, the restriction map from $\Gamma\left(X, \mathcal{O}_{X}\right)$ to $\Gamma\left(S, \mathcal{O}_{X}\right)$ induces by transposition a continuous map from $\widetilde{S}$ to $X$; we denote this map by $\pi$. Clearly, one has $\pi \circ \xi_{S}=$ $j_{S}$, where $j_{S}: S \rightarrow X$ is the inclusion map. Pictorially, we get the following commutative diagram:

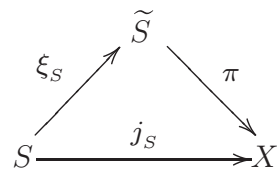

Obviously, $S \subseteq \pi(\widetilde{S})$ with equality when $\xi_{S}$ is surjective.

Notice that if $X$ is non-singular and $S$ is open, then $\pi$ results "locally biholomorphic". However, this fails if $X$ admits singularities. An example is as follows. Take $X$ as the Segre cone in $\mathbb{C}^{4}, X=\left\{z \in \mathbb{C}^{4} ; z_{1} z_{4}=z_{2} z_{3}\right\}$ and $\Sigma=\left\{z \in X ; z_{1}=z_{2}=0\right\}$. Then $X$ is a normal connected three-dimensional Stein space with only one singularity at the origin. The holomorphic map $\pi: \mathbb{C}^{3} \rightarrow X$, $\pi(t, u, v)=(u, v, t u, t v)$, induces a biholomorphism between $\mathbb{C} \times\left(\mathbb{C}^{2} \backslash\{(0,0)\}\right)$ and $W:=X \backslash \Sigma$. Note that $\mathcal{O}(W)$ is a Stein algebra and $\widetilde{W}=\mathbb{C}^{3}$ and $\pi: W \rightarrow X$ is non-locally biholomorphic (non-even an open map).

\section{A few facts about natural sets}

Let $\left(X, \mathcal{O}_{X}\right)$ be a complex space and $K \subset X$ be a compact set. As a consequence of Proposition 2.3, $K$ is natural with respect to the reduced complex structure on 
$X$ if and only if $K$ is natural with respect to the any infinitesimal extension of its reduced structure. Therefore $K$ is structurally acyclic with respect to $\mathcal{O}_{X}$ if and only if $K$ is structurally acyclic with respect to the reduced structure $\overline{\mathcal{O}}_{X}$.

One simple question that comes immediately concerns the hereditary property of natural sets. In this area we have:

Proposition 3.1. Let $Y$ be a reduced Stein space and $X$ be a closed analytic subset of $Y$. Let $K \subset X$ be a compact set. The following hold:

a) If $K$ is natural in $Y$, then $K$ is natural in $X$;

b) If $K$ is natural in $X$ and the restriction map

$$
\rho: \Gamma\left(K, \mathcal{O}_{Y}\right) \rightarrow \Gamma\left(K, \mathcal{O}_{X}\right)
$$

has dense image, then $K$ is natural in $Y$.

Remark 3.2. Part a) is known as Rickart's theorem, and b) is a particular case of a more general statement [36, Theorem 5.14, page 365]. This proposition suggests the interesting question whether being natural is independent of the complex representative.

On the other hand, we would like the notion "natural compact set" to be universal. However, it is non-clear whether or not the condition on the density of the range of $\rho$ is, in general, superfluous. In particular we do non-know the answer to the following question:

Let $X$ be an arbitrary analytic hypersurface of $\mathbb{C}^{n}$, for $n \geq 3$. Is every natural compact set $K$ in $X$ also natural in $\mathbb{C}^{n}$ ?

Remark 3.3. The density of the range of $\rho$ holds true ( $\rho$ is even surjective), for instance, when $K$ is a Stein compact set or if there is a holomorphic retraction from $Y$ onto $X$ (e.g. if $X$ and $Y$ are smooth).

The subsequent proposition improves [36, Theorem 5.17], where the "only if" part is considered.

Proposition 3.4. Let $\pi: X \rightarrow Y$ be a finite holomorphic surjection of Stein spaces. Let $K \subset Y$ be a compact set. Then $K$ is natural in $Y$ if and only if $\pi^{-1}(K)$ is natural in $X$.

Proof (Sketch). Clearly we may assume that $X$ and $Y$ are of finite dimension. Notice that the "only if" part is clear using Theorem 2.2. To deal with the "if" implication one proceeds by induction on $n:=\operatorname{dim}(Y)$ assuming that the theorem is true for complex spaces of dimension less than $n$ and by standard arguments we reduce ourselves to prove the theorem when

i) either $Y$ is normal or

ii) $X$ is the normalization of $Y$.

But this results after a careful analysis of the arguments presented in [38] so we omit the proof. 
Corollary 3.5. Let $X$ be a Stein space and let $\left\{X_{i}\right\}_{i}$ be the decomposition of $X$ into its irreducible components. Then a compact set $K \subset X$ is natural in $X$ if and only if for each index $i, K \cap X_{i}$ is natural in $X_{i}$.

Corollary 3.6. Let $X$ be a Stein manifold. Then a compact set $K$ in $X$ is natural in $X$ if and only if $K$ has a nonschlicht Stein neighborhood basis.

This result is due to Birtel [5] for $X=\mathbb{C}^{n}$. (Observe that it is necessary to introduce Riemann domains here, because it is non-true that a natural set is necessarily an intersection of Stein open sets. Examples have been given in [7] and [30].)

We can avoid the use of Proposition 3.1 and Remark 3.3 and reduce ourselves instead to Birtel's theorem. We do this in four steps whose straightforward proofs are omitted (simple exercices that are left to the interested reader).

I) Let $X$ be a complex submanifold of a Stein manifold $\Omega$ such that there is a holomorphic retraction $\rho: \Omega \rightarrow X$ with connected fibers. Then, for every open set $U$ in $X$, if $\sigma: \tilde{W} \rightarrow W$ is the envelope of holomorphy of $W:=$ $\rho^{-1}(U)$, then $(\rho \circ \sigma)^{-1}(X) \rightarrow X$ is the envelope of holomorphy of $U$.

II) Let $X$ be a Stein manifold and $\pi: E \rightarrow X$ be a holomorphic vector bundle over $X$. Let $U$ be an open set in $X$ and $\sigma: \widetilde{U} \rightarrow X$ be the envelope of holomorphy of $U$. Then in the following Cartesian square,

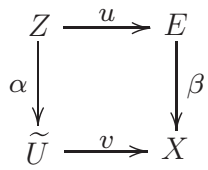

$Z$ is the envelope of holomorphy of $\pi^{-1}(U)$.

III) Let $\pi: X \rightarrow Y$ be a holomorphic map between complex spaces such that the induced map $\pi^{\star}: \mathcal{O}(Y) \rightarrow \mathcal{O}(X)$ is surjective. Then, for all compact set $L$ in $Y$ there is a compact set $F$ in $X$ and a positive constant $C$ such that, for every holomorphic functions $f \in \mathcal{O}(X)$ and $g \in \mathcal{O}(Y)$ with $\pi^{\star}(g)=f$ one has

$$
\|g\|_{L} \leq C\|f\|_{K} .
$$

IV) Let $X$ be a Stein manifold and $\pi: E \rightarrow X$ be a holomorphic vector bundle over $X$. We regard $X$ as the zero section of $E$ and consider a continuous plurisubharmonic function $\Phi: E \rightarrow[0, \infty)$ with $\{\Phi=0\}=X$ (for instance one may take $\Phi(v)=\|v\|^{2}$ with respect to a suitable hermitian metric on $E$ ).

Then, for an open set $U \subset X$ and constant $C>0$, if $\sigma: \widetilde{U} \rightarrow X$ is the envelope of holomorphy of $U$ and $\Omega=\{v \in E ; \Phi(v)<C\}$, then in the following cartesian square

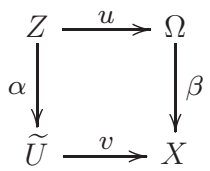

$Z$ is the envelope of holomorphy of $\pi^{-1}(U) \cap \Omega$. 


\section{Proof of Theorem 1.2}

We shall prove a more general version than that stated and in order to do this we introduce the following definition. A space $\left(S, \mathcal{O}_{S}\right)$ in $\mathbf{R s}$ is nowhere degenerate if for every point $s_{0} \in S$ there are finitely many sections $\sigma_{1}, \ldots, \sigma_{r}$ ( $r$ might depend on $\left.s_{0}\right)$ in $\Gamma\left(S, \mathcal{O}_{S}\right)$ such that $s_{0}$ lies isolated in the set

$$
\left\{\sigma_{1}=\sigma_{1}\left(s_{0}\right), \ldots, \sigma_{r}=\sigma_{r}\left(s_{0}\right)\right\} .
$$

In this setting, Theorem 1.2 is a consequence of the following Theorem 4.3 for which proof we prepare:

Lemma 4.1. If a compact space $\left(K, \mathcal{O}_{K}\right)$ in $\mathbf{R s}$ is nowhere degenerate, then it may be defined by a complex space $\left(X, \mathcal{O}_{X}\right)$ that is holomorphically spreadable.

The proof of this readily follows using the compacity of $K$ and the following known statement about complex spaces [11]:

Proposition 4.2. Let $f: Y \rightarrow Z$ be a holomorphic map between complex spaces and $k$ be a non-negative integer. Then the subset $\Sigma_{k}(f)$ of $Y$ given by

$$
\Sigma_{k}(f):=\left\{y \in Y ; \operatorname{dim}_{y} f^{-1}(f(y)) \geq k\right\}
$$

is analytic.

Theorem 4.3. Let $\left(K, \mathcal{O}_{K}\right)$ be a compact space in $\mathbf{R s}$ and $\left(X, \mathcal{O}_{X}\right)$ be a complex representative. Assume that $\left(K, \mathcal{O}_{K}\right)$ is nowhere degenerate and structurally acyclic. Then $\left(K, \mathcal{O}_{K}\right)$ is natural in $\left(X, \mathcal{O}_{X}\right)$.

Proof. This is a straightforward consequence of the following two facts.

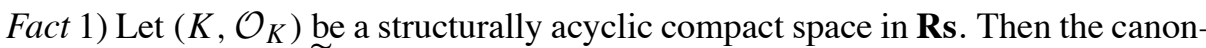
ical map $\xi_{K}: K \rightarrow \widetilde{K}$ is surjective.

For this, assume in order to reach a contradiction that there is $\chi \in \widetilde{K}$ which does non-belong to the image of $\xi_{K}$. Therefore, for each point $x \in K$ there is $f \in$ $\mathcal{O}(K)$ so that $f(x) \neq 0$ and $f(\chi)=0$. By compactness of $K$, there exist functions $f_{1}, \ldots, f_{r} \in \mathcal{O}(K)$ such that $f_{j}(\chi)=0, j=1, \ldots, r$, and these functions do nonvanish simultaneously at some point in $K$ so that there is an open neighborhood $W$ of $K$ on which $f_{j}^{\prime} s$ do non-have common zeros. This means that $\mu: \mathcal{O}^{r} \rightarrow \mathcal{O}$ defined by $\mu\left(h_{1}, \cdots, h_{r}\right):=\sum_{j} f_{j} h_{j}$ is surjective on an open neighborhood $W$ of $K$ (on which the $f_{j}$ 's do non-have common zeroes). Now consider on $W$ the Koszul sequence

$$
0 \rightarrow \Lambda^{0} \mathcal{O}_{X}^{r} \rightarrow \cdots \rightarrow \Lambda^{i} \mathcal{O}_{X}^{r} \rightarrow \Lambda^{i+1} \mathcal{O}_{X}^{r} \rightarrow \cdots \rightarrow \Lambda^{r} \mathcal{O}_{X}^{r} \simeq \mathcal{O}_{X}
$$

By splitting it into short exact sequences, the hypothesis implies readily that $H^{1}(K, \mathcal{R})=0$, where $\mathcal{R}=\operatorname{Ker} \mu$. Therefore $\mu: \mathcal{O}^{r}(K) \rightarrow \mathcal{O}(K)$ is surjective; in particular there do exist $h_{1}, \ldots, h_{r} \in \mathcal{O}(K)$ such that $1=\sum_{j} f_{j} h_{j}$. But this is impossible since $1=1(\chi)=\sum_{j} f_{j}(\chi) h_{j}(\chi)=0$. 
Fact 2) Let $\left(K, \mathcal{O}_{K}\right)$ be a structurally acyclic compact space in Rs admitting a holomorphically spreadable complex space $\left(X, \mathcal{O}_{X}\right)$ as representative. Then there is an open set $U$ of $K$ in $X$ such that $\Gamma\left(U, \mathcal{O}_{X}\right)$ separates points of $U$.

The injectivity of $\xi_{K}$ follows easily if we prove that, for each pair of points $x, y \in K, x \neq y$, there exists a holomorphic function $f$ on $K$ such that $f(x) \neq$ $f(y)$.

This is done by induction over $n=\operatorname{dim}(X)$ as follows. Consider the singular sets $\mathrm{S}_{d}$ of $\mathcal{O}_{X}$, which are defined as follows. For any non-negative integer $d$ we let

$$
\mathrm{S}_{d}:=\left\{x \in X ; \operatorname{prof}\left(\mathcal{O}_{X, x}\right) \leq d\right\} .
$$

Thanks to [34, Theorem 1.11], each $\mathrm{S}_{d}$ is an analytic subset of $X$ of dimension $\leq d$. Their importance lie in [34, Corollary 1.11 ] namely, for a holomorphic function $f$ on $X$ the following two conditions are equivalent:

- For each $x \in X$, the germ $f_{x}$ is not a zero divisor in $\mathcal{O}_{X, x}$.

- If $Z_{f}$ denotes the zero set of $f$, then one has $\operatorname{dim}\left(Z_{f} \cap S_{k+1}\right) \leq k$ for all integers $k \geq-1$.

Let $S_{i}^{\prime}$ be the set of isolated points of $S_{i}\left(S_{0}^{\prime}=S_{0}\right)$ and consider $\left\{\Gamma_{j}\right\}_{j \in J}$ be the set of positive dimensional irreducible components of all sets $S_{1}, S_{2}, \ldots$ ( $J$ is at most a countable set of indices). The family $\left\{\Gamma_{j}\right\}_{j \in J}$ is locally finite and $T$, which equals the union of all $S_{i}^{\prime}$ is discrete in $X$. Now, by using Baire's theorem, since $X$ is holomorphically spreadable, there is a holomorphic function $h$ on $X$ such that, for any $j \in J,\left.h\right|_{\Gamma_{j}}$ is not a constant function. Therefore, for $x \in X \backslash T$ and $c \in \mathbb{C}$, $h_{x}-c$ is not a zero-divisor in $\mathcal{O}_{X, x}$. Thus the kernel of the morphims induced by multiplication by $h-c, \mathcal{O}_{X} \rightarrow \mathcal{O}_{X}$ is supported in a discrete set.

Now, if $h(x) \neq h(y)$ we are done. Otherwise, let $c=h(x)=h(y)$. Let $X^{\prime}:=h^{-1}(c)$ viewed as a complex space endowed with the possible non-reduced structure (in fact, the full fiber of $h$ over $c$ ); $\operatorname{dim}\left(X^{\prime}\right)<n$. For $K^{\prime}:=K \cap X^{\prime}$ one checks that $H^{i}\left(K^{\prime}, \mathcal{O}\right)=0$ for all positive integers $i$. Thus, there is a holomorphic function $f^{\prime}$ on $K^{\prime}$ with $f^{\prime}(x) \neq f^{\prime}(y)$. We show that $f^{\prime}$ is, in fact, the restriction of a holomorphic function $f$ on $K$. Since the kernel of the epimorphism $\mathcal{O}_{X} \rightarrow(h-c)$ is supported in a discrete set, it follows that $H^{1}(K,(h-c))=0$. Thus the restriction map $H^{0}(K, \mathcal{O}) \rightarrow H^{0}\left(K^{\prime}, \mathcal{O}\right)$ is surjective, whence the proof of the inductive step. Thus $\xi_{K}$ is injective, as desired.

Remark 4.4. Here we note that in the case when $\mathcal{O}(X)$ gives local coordinates one may choose $U$ such that $\mathcal{O}(U)$ separates points and $U$ is biholomorphic to an analytic subset of an open set in some $\mathbb{C}^{N}$.

\section{Domains of holomorphy}

Let $X$ be a normal Stein space of pure dimension and $D$ be an open set in $X$. If $D$ is Stein, then $D$ is a domain of holomorphy. The converse is valid when $X$ is a 
manifold. However, this is not the case in general, as was pointed out by Grauert and Remmert [14] and [15]. They gave an example of a non-Stein domain of holomorphy in a Stein space (the Segre cone). As a matter of fact, this is generalized in [19] as follows:

Proposition 5.1. Let $X$ be a connected normal Stein space and $\Sigma \subset X$ be a hypersurface (analytic subset of pure codimension one). Then $X \backslash \Sigma$ is a domain of holomorphy in $X$.

The problem whether a domain of holomorphy is itself Stein is related to the Levi problem, which asks whether a domain in $X$ is Stein if it is locally Stein (at all boundary points). Notice that a domain of holomorphy is locally Stein at the boundary points which are non-singular points of $X$.

Below we collect a few facts that can be deduced from [12].

Proposition 5.2. Let $D$ be a domain of holomorphy in $X$. Then for every analytic set $A \subset X$ of codimension at least 2 , the set $\partial D \backslash A$ is dense in $\partial D$.

Proof. Indeed, assume, in order to reach a contradiction, that $\partial D \backslash A$ is non-dense in $\partial D$. Thus there is $a \in A \cap \partial D$ and a connected open neighborhood $U$ of $a$ such that $U \cap \partial D \subset A$. Now as $U \backslash A$ is connected and does non-meet $\partial D$, either $U \backslash A \subset D$ or $U \backslash A \subset X \backslash \bar{D}$. But the latter possibility cannot hold as it gives that $U \cap \bar{D} \subset A$ which is non-true since $A$ is rare (hence has empty interior). Therefore $U \backslash A \subset D$. Further, as $X$ is normal and codim $A \geq 2$, the restriction map $\mathcal{O}(U) \rightarrow \mathcal{O}(U \backslash A)$ is bijective and this contradicts easily the hypothesis that $D$ is a domain of holomorphy.

Proposition 5.3. Let $D$ be relatively compact in $X$. Assume that $D$ is locally Stein at $\partial D \backslash \operatorname{Sing}(X)$. Then, for every sequence $\left\{x_{n}\right\}_{n} \subset D \backslash \operatorname{Sing}(X)$ with at most finitely many accumulation points each of which being in $\partial D \backslash \operatorname{Sing}(X)$, if $\left\{c_{n}\right\}$ is an arbitrary sequence of complex numbers, then there is a holomorphic function $f$ on $D$ such that $f\left(x_{n}\right)=c_{n}$ for all $n$.

Proof. Let $b_{1}, \ldots, b_{k}$ be the accumulation points of $\left\{x_{n}\right\}_{n}$. From [12] it follows that for each $k$ there is a holomorphic map $g_{k}: D \rightarrow \mathbb{C}^{v_{k}}$ on $X$ such that $\left\|g_{k}(x)\right\|$ tends to infinity as $x \in D \backslash \operatorname{Sing}(X)$ tends to $b_{k}$. This implies readily that we can produce a holomorphic mapping $h: D \rightarrow \mathbb{C}^{q}$ such that $h(\Gamma)$ is discrete in $\mathbb{C}^{q}$. Now select $f: X \rightarrow \mathbb{C}^{p}$ holomorphic such that $\left.f\right|_{D}$ is injective. It follows that the holomorphic map $(f, h)$ on $D$ sends $\Gamma$ into a discrete set of $\mathbb{C}^{p+q}$, whence the assertion by a standard argument.

Remark 5.4. Let $X$ be a Stein manifold and $D \subset X$ an open set. Assume that there is a rare analytic set $A$ in $X$ such that $D$ is locally Stein on $\partial D \backslash A$ and $\partial D \backslash A$ is dense in $\partial D$. Then $D$ is Stein.

Proposition 5.5. A relatively compact domain $D$ in $X$ is a domain of holomorphy if and only if $D$ is locally Stein at every point of $\partial D \backslash \operatorname{Sing}(X)$ and $\partial D \backslash \operatorname{Sing}(X)$ is dense in $\partial D$. 
Remark 5.6. In the above assertion one can replace $\operatorname{Sing}(X)$ by any rare analytic subset of $X$.

Corollary 5.7. For a finite surjective holomorphic map $\pi: Y \rightarrow X$ between Stein normal complex spaces, a relatively compact open subset $D$ of $X$ is a domain of holomorphy in $X$ if and only if $\pi^{-1}(D)$ is a domain of holomorphy in $Y$.

Proposition 5.8. Let $D$ be a relatively compact domain of holomorphy in a normal Stein surface $X$. Then $D$ is Stein if and only if $\mathcal{O}(D)$ is a Stein algebra.

Proof. We deal with the "if"part. This condition means that there is a normal Stein surface $\widetilde{D}$, a holomorphic open immersion $\iota: D \rightarrow \widetilde{D}$ and a holomorphic map $\pi: \widetilde{D} \rightarrow X$ which stay in a commutative diagram

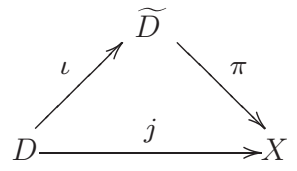

From [9] and Proposition 8, it follows that $\widetilde{D}=\iota(D) \cup \pi^{-1}(\operatorname{Sing}(X))$ and $\pi^{-1}(\operatorname{Sing}(X))$ is discrete. Thus $\iota(D)=\widetilde{D} \backslash T$ for some discrete subset of $\widetilde{D}$ with $\pi(T) \subset \partial D$. If there is a point $\zeta_{0} \in \widetilde{D} \backslash \iota(D)$ and as $\pi$ has discrete fibers, there are relatively compact open neighborhoods $V$ and $W$ of $\zeta_{0}$ and $\pi\left(\zeta_{0}\right)$, respectively, such that $\pi$ induces a finite map $\rho: V \rightarrow W$ and $W$ is Stein and connected. Thus $\rho$ is surjective so that $W \backslash\left\{\pi\left(\zeta_{0}\right)\right\} \subset D$ showing that $D$ is not a domain of holomorphy.

Remark 5.9. This statement fails for $X$ of dimension at least 3 as the example of Grauert and Remmert considered in Section 3 shows.

Now we discuss domains of holomorphy in non-necessarily normal Stein spaces. Let $X$ be a reduced Stein space and $D$ be an open set in $X$. First, we give an example that explains why the ordinary definition cannot be translated $a d$ litteram to the non-normal (even irreducible) complex spaces.

Take $X:=\left\{(z, w) \in \mathbb{C}^{2} ; z w=0\right\}$. Obviously $X$ is a Stein curve. Let $a, b \in \mathbb{R}$, with $0<a<b$. Set $\Omega:=\{(z, w) \in X ; \max (|z|,|w|)<b\}$. Then $\Omega$ is a Stein open subset of $X$.

Let $U_{1}=\{(z, w) \in X ; a<|z|<b\}$ and $U_{2}=\{(z, w) \in X ;|z|<b\}$. These are connected, non-empty open subsets of $X$ with $U_{1} \subset U_{2} \cap \Omega \neq U_{2}$ and it is easily seen that, for every $f \in \mathcal{O}(\Omega)$ there exists $f_{2} \in \mathcal{O}\left(U_{2}\right)$ with $f=f_{2}$ on $U_{1}$. Thus $\Omega$ will not be a domain of holomorphy with the standard definition from the smooth case. (This example can be "adjusted" in order to get $X$ irreducible as follows. Start with $X=\left\{(z, w) \in \mathbb{C}^{2} ; w^{2}=z^{2}+z^{3}\right\}$; let $\pi: \mathbb{C} \rightarrow X$ be the normalization map which is given by

$$
\pi(t)=\left(t^{2}-1, t\left(t^{2}-1\right)\right), \quad t \in \mathbb{C} .
$$


Then $X$ is irreducible but non-locally irreducible (at its only singular point, namely $0)$; note that $\pi^{-1}(0)=\{-1,1\}$. Now, we can repeat the idea from above. Let $V_{1}=\{t \in \mathbb{C} ;|t+1|<\epsilon\}$ and $V_{2}=\{t \in \mathbb{C} ;|t-1|<\epsilon\}$ for $\epsilon>0$ sufficiently small (such that $\left.\pi\left(\bar{V}_{1}\right) \cap \pi\left(\bar{V}_{2}\right)=\{0\}\right)$. Set $\Omega:=\pi\left(V_{1} \cup V_{2}\right)$. Then $\Omega \subset X$ is open and $\pi\left(V_{1}\right), \pi\left(V_{2}\right)$ are its two irreducible components that meet only at $0 \in X$. Similarly as above it is straightforward to verify that, although $\Omega$ is Stein, it fails to be a domain of holomorphy according to the ordinary definition as in the smooth case.)

The correct intrinsic notion that fits our purpose and naturally extends that from the normal ambiance setting is:

Definition 5.10. Let $X$ be a reduced complex space of pure dimension. An open set $D$ in $X$ is said to be a domain of holomorphy if there does not exist non-empty open sets $U_{1}$ and $\widehat{U}_{2}$ of $X$ and an irreducible component $Y$ of $X$ such that:

a) the analytic set $U_{2}:=\widehat{U}_{2} \cap Y$ is irreducible,

b) one has $U_{1} \subset U_{2} \cap D$ and $U_{2} \not \subset D$, and

c) for every $f \in \mathcal{O}(D)$ there exists $f_{2} \in \mathcal{O}\left(U_{2}\right)$ with $f=f_{2}$ on $U_{1}$.

Clearly, if $X$ is normal, we recover the standard definition of domain of holomorphy. A class of examples is obtained as follows.

Proposition 5.11. Let $X$ be a reduced Stein space and $A$ be the set of the nonnormal points of $X$. Suppose that $A$ is discrete and non-empty. Then $D:=X \backslash A$ is a domain of holomorphy in $X$.

Proof. Let $a \in A$ and $U$ an open neighborhood of $a$ with $U \cap A=\{a\}$. The Mayer-Vietoris sequence and standard arguments give that the restriction $\mathcal{O}(X) \rightarrow$ $\mathcal{O}(X \backslash\{a\})$ is surjective if and only if $\mathcal{O}(U) \rightarrow \mathcal{O}(U \backslash\{a\})$ is surjective. Now let $A=\left\{a_{k}\right\}_{k}$ and for each $k$ there is $\left.f_{k} \in \mathcal{O}\left(X \backslash a_{k}\right\}\right)$ which cannot extend over $a_{k}$ Then $f:=\sum \epsilon_{k} f_{k}$ for $\left\{\epsilon_{k}\right\}_{k}$ a sequence of positive numbers that decreases rapidly to zero, shows that $D$ is a domain of holomorphy.

Remark 5.12. As a matter of fact, the non-extension property is a way to show that certain complex spaces are non-normal. For instance, we give subsequently two examples of non-Stein domains of holomorphy in globally irreducible and locally irreducible Stein surfaces.

The first one is inspired by the above Segre cone and it is as follows. Let $W=\left\{\left(z_{1}, z_{2}, z_{3}, z_{4}\right) \in \mathbb{C}^{4} ; z_{1}=s t, z_{2}=s, z_{3}=s t^{3}, z_{4}=s t^{4}, s, t \in \mathbb{C}\right\}$. Take $X:=\bar{W}$, the closure of $W$ in $\mathbb{C}^{4}$, which equals $W$ together with the complex line in the fourth $z_{4}$ direction; $X$ is a two dimensional analytic subset of $\mathbb{C}^{4}$ and is locally irreducible at every point but fails to be normal only at 0 . (Because $\operatorname{Sing}(X)=\{0\}$, and, since $W \backslash\{0\}=X \backslash \mathbb{C}_{z_{4}} \subset \operatorname{Reg}(X) \subset \bar{W}$ it follows that $X$ and $\operatorname{Reg}(X)=X \backslash\{0\}$ are connected so that $X$ is globally irreducible. Similarly we show that $X$ is locally irreducible at 0 . For if $A$ is a connected subset of a topological space $T$, then any set $B$ in $T$ with $A \subset B \subset \bar{A}$ is connected.) 
The function $f=z_{1}^{2} / z_{2}: X \backslash\{0\} \rightarrow \mathbb{C}$ is holomorphic but cannot be extended holomorphically to the whole space $X$. (Since $X=W \cup \mathbb{C}_{z 4}$, the function $\tilde{f}: X \rightarrow$ $\mathbb{C}$ given by $z_{1}^{2} / z_{2}$ for $z_{2} \neq 0$ and 0 for $z_{2}=0$ is continuous on $X$, holomorphic on $X \backslash\{0\}$ but does non-extends holomorphically to $X$, as it can be easily seen using Taylor series developpment around the origin!) Thus $D:=X \backslash\{0\}$ is a domain of holomorphy in $X$. On the other hand, if $v: X^{\star} \rightarrow X$ is the normalization map, then $v$ is a topological homeomorphism and $D^{\star}:=v^{-1}(D)$, which equals $X^{\star}$ with a point deleted, is not a domain of holomorphy in $X^{\star}$.

For the second example we employ the Stein surface $X$ in $\mathbb{C}^{2}$ due to Harvey that is given as the image of the proper holomorphic mapping

$$
h: \mathbb{C}^{2} \rightarrow \mathbb{C}^{4},(z, w) \mapsto\left(z^{2}, z^{3}, w, z w\right) .
$$

It is straightforward to check that $X$ has only one singularity at the origin and $X$ is globally irreducible, locally irreducible, and the map $h$ is a homeomorphism onto $X$, so that $h$ is the normalization of $X$.

Now we claim that $D:=X \backslash\{0\}$ is a domain of holomorphy in $X$ and its preimage $h^{-1}(D)=\mathbb{C}^{2} \backslash\{(0,0)\}$ is not a domain of holomorphy in $\mathbb{C}^{2}$.

But this follows because the function $f: D \rightarrow \mathbb{C}$ given by $f(\zeta)=z_{2} / z_{1}$ if $z_{1} \neq 0$ and $z_{4} / z_{3}$ if $z_{3} \neq 0$, which is holomorphic on $X \backslash\{0\}$, does non-extend holomorphically to the whole space $X$. (Otherwise, the holomorphic function $X \rightarrow$ $\mathbb{C}^{2}, z=\left(z_{1}, z_{2}, z_{3}, z_{4}\right) \mapsto\left(f(z), z_{3}\right)$ would be an inverse for $h: \mathbb{C}^{2} \rightarrow X$ which is absurd since $X$ is non-smooth!)

Remark 5.13. The above examples show that for a domain of holomorphy $D$ in an irreducible complex space $X$, its preimage $D^{\star}$ via the normalization map $X^{\star} \rightarrow X$, in general, fails to be a domain of holomorphy in $X^{\star}$. Note that, if we choose $D=W \backslash\{0\}$ where $W$ is a relatively compact connected neighborhood of 0 , then $\mathcal{O}(D)$ is a Stein algebra (isomorphic to $\mathcal{O}\left(h^{-1}(W)\right)$ ) so that the analogous assertion of Proposition 5.8 fails when the ambient space is non-normal.

In this circle of ideas, let us mention the following interesting question (see also [13]) that originates in Grauert and Remmert's papers [14,15]:

Is it true that in any normal Stein surface any domain of holomorphy is Stein?

Theorem 5.14. Let $\pi: X^{\star} \rightarrow X$ be the normalization map of a pure dimensional Stein space $X$. Let $D$ be a relatively compact open subset of $X$. Then $D$ is a domain of holomorphy in $X$ provided that $D^{\star}:=\pi^{-1}(D)$ is a domain of holomorphy in $X^{\star}$.

Proof. We split the proof into three steps:

Step 1) The set $\Lambda:=\partial D \backslash \pi\left(\operatorname{Sing}\left(X^{\star}\right)\right)$ is dense in $\partial D$.

This is a consequence of a simple topological fact stating that for a proper surjective mapping $\varphi: S \rightarrow T$ between Hausdorff topological spaces $S$ and $T$, for every open set $D \subset T, \varphi(\partial \Omega)=\partial D$, where $\Omega=\varphi^{-1}(D)$ and the observation that the set $B:=\pi\left(\operatorname{Sing}\left(X^{\star}\right)\right)$ has codimension $\geq 2$ (because $X$ has pure dimension), hence $\pi^{-1}(B)$ has codimension $\geq 2$, so that $\partial D^{\star} \backslash \pi^{-1}(B)$ is dense in $\partial D^{\star}$. 
Step 2) For any point $a \in \Lambda \cap \partial D$ and any sequence of distinct points $\left\{x_{v}\right\}_{v}$ in $\operatorname{Reg}(D)$ converging to $a$ and any sequence $\left\{\lambda_{\nu}\right\}_{\nu}$ of complex numbers there is a holomorphic function $f$ on $D$ such that $f\left(x_{v}\right)=\lambda_{v}$ for all $v$.

To see this, first observe that as $X$ is Stein of finite dimension, then for every coherent sheaf of ideals $\mathcal{I}$ there are finitely many holomorphic functions $h_{1}, \ldots, h_{m} \in$ $\Gamma(X, \mathcal{I})$ whose common zeros set equals the vanishing set of $\mathcal{I}$.

We apply this for $\mathcal{I}$ the ideal sheaf of universal denominators of $\widetilde{\mathcal{O}}_{X}$; its vanishing set is the non-normal locus $X^{\prime}$ of $X$. Here $\widetilde{\mathcal{O}}_{X}$ is the coherent sheaf of germs of weakly holomorphic functions on $X$. Note that $\widetilde{\mathcal{O}}_{X}=\pi_{\star}\left(\mathcal{O}_{X^{\star}}\right)$ and for $h \in \Gamma(X, \mathcal{I})$ and $g \in \Gamma\left(X, \widetilde{\mathcal{O}}_{X}\right)$ one has $h g \in \Gamma\left(X, \mathcal{O}_{X}\right)$.

Now, for each $v$ consider $y_{v} \in X^{\star}$ such that $\pi\left(y_{v}\right)=x_{v}$. Let $\pi^{-1}(a)=$ $\left\{b_{1}, \ldots, b_{r}\right\}$. Clearly $b_{1}, \ldots, b_{r}$ belong to $\operatorname{Reg}\left(X^{\star}\right)$.

Consider first $r=1$. We produce a holomorphic function $f$ on $D$ of the form $f=\sum_{i=1}^{m} h_{i} g_{i}$ for suitable $g_{i} \in \widetilde{\mathcal{O}}_{X}(D)$ given in the following way. For each index $i, 1 \leq i \leq m$, we let $T_{i}:=\left\{v \in \mathbb{N} ; h_{i}\left(x_{v}\right) \neq 0\right\}$. Clearly $\cup_{i} T_{i}=\mathbb{N}$. Select complex numbers $\gamma_{i v}$ such that $\sum_{i=1}^{m} \gamma_{i v} h_{i}\left(x_{v}\right)=1$ for all $v$. Now from Proposition 5.3 there are $g_{i} \in \widetilde{\mathcal{O}}_{X}(D)$ such that $g_{i}\left(x_{v}\right)=\lambda_{\nu} \gamma_{i v}$ if $\nu \in T_{i}$ and 0 otherwise. It follows that $f\left(x_{v}\right)=\lambda_{\nu}$ for all $v$, as desired.

In case $r>1$ we partitionate $\mathbb{N}$ into finitely many sets $I_{1}, \ldots, I_{r}$ such that, for each index $s, 1 \leq s \leq r$, the sequence $\left\{y_{\nu}\right\}_{v \in I_{s}}$ converges to $b_{s}$. By the above discussion there are holomorphic functions $f_{1}, \ldots, f_{r}$ on $D$ such that $f_{s}\left(x_{v}\right)=\lambda_{v}$ if $v \in I_{S}$ and 0 otherwise. Then $f:=\sum_{i=1}^{r} f_{i}$ fulfils the said property.

Step 3) End of proof of theorem. Assume, in order to reach a contradiction that $D$ is not a domain of holomorphy; thus there are open sets $U_{1}$ and $U_{2}^{\star}$ of $X$ and an irreducible component $X_{1}$ of $X$ according to Definition 5.10. Let $X_{1}^{\star}$ be the connected component of $X^{\star}$ that projects through $\pi$ onto $X_{1}$.

Since $U_{2}$ is irreducible, $U_{2}^{\star}=\pi^{-1}\left(U_{2}\right)$ is connected. Furthermore, the irreducible components $\left\{A_{i}\right\}_{i}$ of $D \cap U_{2}$ are in one-to-one correspondence with the connected components $\left\{A_{i}^{\star}\right\}_{i}$ of $D^{\star} \cap U_{2}^{\star}$. Let $A_{0}$ be such that $A_{0} \cap U_{1} \neq \emptyset$, hence $A_{0}^{\star} \cap U_{1}^{\star} \neq \varnothing$. Then there is a point $b \in \partial D^{\star} \backslash \operatorname{Sing}\left(X^{\star}\right)$ which is an accumulation point of $A_{0}^{\star} \cap D^{\star} \backslash \operatorname{Sing}\left(X^{\star}\right)$ so that there is a sequence $\left\{x_{\nu}^{\star}\right\}_{\nu}$ of points in $A_{0}^{\star} \cap D^{\star} \backslash \operatorname{Sing}\left(X^{\star}\right)$ converging to $b$. Set $a=\pi(b)$ and $\left.x_{v}=\pi^{(} x_{v}^{\star}\right)$ for all $v$. Thanks to Step 2, there is a holomorphic function $f$ on $D$ which is unbounded on the sequence $\left\{x_{v}\right\}_{v}$. On the other hand, by our assumption, there is a holomorphic function $f_{2}$ on $U_{2}$ such that $f_{2}=f_{1}$ on $U_{1}$, therefore on $A_{0}$, by the identity theorem. Thus the sequence $\left\{f\left(x_{v}\right)\right\}_{v}$ is convergent to $f_{2}(a)$, which is non-possible, whence the theorem.

As a consequence we extended a result due to Fornæss and Narasimhan that is recovered when $X$ is normal, namely:

Corollary 5.15. Let $X$ be a reduced Stein space of pure dimension. Then every relatively compact locally Stein open subset of $X$ is a domain of holomorphy. 
Corollary 5.16. Let $X$ be a reduced Stein space of pure dimension. Let $D$ be a relatively compact open set that is an increasing union of Stein open sets. Then $D$ is a domain of holomorphy in $X$.

Proof. We shall need the following fact from [4, page 365]:

Let $Y$ be a normal Stein space of pure dimension $\geq 2$ and $K \subset Y$ is a holomorphically convex compact set. Then the canonical restriction $\Gamma(Y, \mathcal{O}) \rightarrow$ $\Gamma(Y \backslash K, \mathcal{O})$ is bijective.

This gives readily that in a normal Stein space of pure dimension $\geq 2$ the complement of a holomorphically convex compact set cannot be an increasing union of domains of holomorphy, a fortiori such a complement cannot be a domain of holomorphy.

Now, coming back to the proof of the corollary, thanks to Theorem 5.14, we may assume that $X$ is normal, and, then, the proof amounts to show that $\partial D \backslash$ $\operatorname{Sing}(X)$ is dense in $\partial D$. Arguing by contradiction, then by slicing and normalization (Stein property of open sets is stable under normalization), we arrive at a normal Stein surface together with a point in it for which its complement can be written as an increasing union of Stein open subsets, a setting that is ruled out by the above discussion.

In the circle of ideas presented here one might ask the following question:

Let $X$ be a reduced Stein space of pure dimension and $\left\{D_{i}\right\}_{i \in I}$ be a family of Stein open sets in $X$. Does it follow that $D:=$ the interior of $\cap_{i \in I} D_{i}$ is a domain of holomorphy in $X$ ?

The answer is "No!" and a counterexample is the following. Let $X$ be the union in $\mathbb{C}^{4}$ of two complex planes $A$ and $B$ that intersects only at the origin 0 . Let $B_{v}$ be a decreasing sequence of open Stein sets in $B$ whose intersection is $\{0\}$. Then $D_{v}:=A \times B_{v}$ are as desired since in this case $D=A \backslash\{0\}$.

In the same vein one might ask whether or non-slicing preserves domains of holomorphy:

Let $X$ be a connected normal Stein space of dimension $\geq 3$ and $D$ a domain of holomorphy in $X$. Does it follow that, for any hypersurface $A$ in $X, A \cap D$ is a domain of holomorphy in A?

Again the answer is negative. Take $X=\left\{z \in \mathbb{C}^{4} ; z_{1} z_{2}=z_{3} z_{4}\right\}$ and $D=X \backslash \Sigma$, where $\Sigma=\left\{z \in X ; z_{2}=z_{3}=0\right\}$, so that $X$ is a normal connected space and $D:=$ $X \backslash \Sigma$ is a domain of holomorphy in $X$. However, for $A=\left\{z \in \mathbb{C}^{4} ; z_{1}=z_{4}=0\right\}$, which is a hypersurface in $X$, the open set $D \cap A$ is not a domain of holomorphy in $A$. (If we identify $A$ with $\mathbb{C}^{2}$, then $A \cap D$ becomes $\mathbb{C}^{2} \backslash\{(0,0)\}$.)

An important result that is very useful when slicing domains of holomorphy is the construction of irreducible principal Stein surfaces in Stein spaces. 
Theorem 5.17. Let $X$ be a Stein space of pure dimension $n$. Let $Y \subset X$ be a locally analytic set which is Stein, irreducible and of dimension $n$. Let also $\Lambda$ and $\Lambda^{\prime}$ be two disjoint discrete subsets in $X$. Then, there exists a holomorphic function $f \in \mathcal{O}(X)$ with the following properties:

a) The set $Z_{f}:=\{f=0\}$ contains $\Lambda$ and is disjoint of $\Lambda^{\prime}$.

b) The analytic set $Y \cap Z_{f}$ is irreducible and $f$ has multiplicity one on it.

The proof follows similarly as with its version in [39] so we confine ourselves to point out the necessary changes. For instance, on [39, page 202] the "almostproper" holomorphic map $\pi$ should now be considered from $Y$ into $\mathbb{C}^{n}$. Also there we did non-have a condition with a discrete set $\Lambda^{\prime}$. However, this can be easily fulfilled as we reasoned using Baire's theorem and because $f$ is of the form $f=$ $F_{g}:=\sum_{i=1}^{m} g_{i} h_{i}$, with fixed $h_{1}, \ldots, h_{m} \in \mathcal{O}(X)$ such that $\Lambda=\left\{h_{1}=\cdots=\right.$ $\left.h_{m}=0\right\}$ and $g=\left(g_{1}, \ldots, g_{m}\right)$ runs in a $G_{\delta}$ set. The simple observation that $\left\{g \in \mathcal{O}(X)^{m} ; \exists a \in \Lambda^{\prime}, F_{g}(a) \neq 0\right\}$ is open and dense in $\mathcal{O}(X)^{m}$ concludes the proof of Theorem 5.17.

\section{Proof of Theorem 1.3}

We begin by observing that, thanks to Theorem 2.2, for a compact set $F$ in a Stein space $X$, non-necessarily reduced, the vanishing of all cohomology groups $H^{i}\left(F, \mathcal{O}_{X}\right), i \geq 1$ is equivalent with $H^{i}\left(F, \mathcal{O}_{\operatorname{red} X}\right)=0$ for all $i \geq 1$ (both conditions amounts to saying that $F$ is a natural set).

Now consider the singular sets $S_{d}$ of $\mathcal{O}_{X}, d=0,1, \ldots$. Then we let $S_{d}^{\prime}$ denotes the union of all irreducible components of dimension $d$ of $S_{d}, d=1,2, \ldots$; the union $S^{\prime}$ of all these $S_{d}^{\prime}$ is an analytic set in $X$. Since $X$ is Stein, there is a holomorphic function $f$ on $X$ such that its zero set $Z_{f}$ has pure codimension one and $f$ does non-vanish identically on no positive dimensional irreducible component of some $S_{d}$ for some $d>0$. (As a matter of fact, there is a discrete set $T$ of $X$ which is obtained by selecting points from every positive dimensional irreducible component of all $S_{d}$ with $d>0$ and we require that $Z_{f}$ is disjoint from $S$.) This gives that the $\mathcal{O}_{X}$-endomorphisn induced by multiplication by $f$ is injective. By hypothesis on $K$ we deduce readily that $H^{i}\left(K, f \mathcal{O}_{X}\right)=0$ for $1 \leq i$. This implies immediately that for $K^{\prime}:=K \cap\{f=0\}$ in $X^{\prime}:=f^{-1}(0)$, one has $H^{i}\left(K^{\prime}, \mathcal{O}_{X^{\prime}}\right)=0$ whenever $1 \leq i$. Here $X^{\prime}$ is the possibly non-reduced fibre $f^{-1}(0)$. Granting Theorem 2.2, it follows that $K^{\prime}$ is natural in $X^{\prime}$ endowed with the reduced structure.

To proceed with the proof, consider for simplicity $n=2$. Now, assume, in order to reach a contradiction, that $\Omega:=\operatorname{int}(K)$ is not a domain of holomorphy in $X$; let $U_{1}$ and $U_{2}$ be as in Definition 5.10. Then select points $x_{1} \in U_{1} \backslash \Lambda$ and $x_{2} \in U_{2} \backslash(K \cup T)$. By Theorem 5.17 there is a holomorphic function $f$ on $X$ such that the following properties are satisfied:

- $f\left(x_{1}\right)=f\left(x_{2}\right)=0$ and for every $x \in \Lambda^{\prime}, f(x) \neq 0$.

- The analytic set $U_{2} \cap Y$ is irreducible, where $Z=\{f=0\}$. 
Now, since $K \cap Y$ is natural in $Y$, it follows that every holomorphic function on a neighborhood of $K \cap Y$ in $Y$ extends to a holomorphic function on a neighborhood of $K$ in $X$. Let $g$ be a holomorphic function on $Y$ such that $\{g=0\}=\left\{x_{2}\right\}$. (In fact, since $Y$ is a Stein curve, one has $H^{2}(Y, \mathbb{Z})=0$ so that it results $H^{1}\left(Y, \mathcal{O}_{Y}^{*}\right)=0$. Thus every multiplicative Cousin problem on $Y$ is solvable.)

There is a holomorphic function $G$ on a neighborhood $W$ of $K$ in $X$ such that $\left.G\right|_{W \cap Y}$ equals $1 / g$ on a neighborhood of $K \cap Y$ in $Y$. Moreover, there is a holomorphic function $G_{2}$ on $U_{2}$ which equals $G$ on $U_{1}$. The identity theorem for holomorphic functions and since $U_{2} \cap Y$ is irreducible imply that $\left.g \cdot G_{2}\right|_{U_{2} \cap Y}-1$ vanishes identically on $U_{2} \cap Y$ which is the desired contradiction since $g\left(x_{2}\right)=0$.

Eventually, the general case concludes easily by standard reccurent arguments applying Theorem 5.17.

In the circle of ideas dealt with in this article, we raise the following question:

Let $X$ be a normal Stein surface. Does it follow that, for any compact set $K \subset X$ such that $H^{1}(K, \mathcal{O})=0$, the interior of $K$ is Stein?

\section{A final remark}

Granting the cohomological characterizations of both natural sets as well as that of domains of holomorphy in $\mathbb{C}^{n}$ one is led to think that there is a close relation between these two notions; however, it is not so because, in general, the closure of a Stein open set in $\mathbb{C}^{n}(n \geq 2)$ may fail to be natural. The simplest example is the Hartogs triangle $\left\{(z, w) \in \mathbb{C}^{2} ;|z|<|w|<1\right\}$.

Subsequently we give a non-vanishing result, which might be well-known but we were unable to find it explicitely in the literature, namely:

Proposition 7.1. Let $K$ be a compact set in $\mathbb{C}^{n}$ such that there is a Hartogs pair of order $q,\left(\widehat{W}_{q}, W_{q}\right)$ such that $\bar{W}_{q} \subset K$ and $\widehat{W}_{q} \not \subset K$. Then $H^{q}(K, \mathcal{O})$ does non-vanish. Moreover, the cohomology group $H^{q}(K, \mathcal{O})$ has infinite dimension as a complex vector space.

Before proceeding to the proof, let us recall a bit of terminology. A simple Hartogs figure of order $q$ in $\mathbb{C}^{n}, 1 \leq q<n$, is given as follows. Write $n=q+k$ and set

$$
\Omega_{q}:=\left(\left(\Delta^{q}\left(r_{1}\right) \backslash \bar{\Delta}^{q}\left(r_{2}\right)\right) \times \Delta^{k}\left(s_{1}\right)\right) \cup\left(\Delta^{q}\left(r_{1}\right) \times \Delta^{k}\left(s_{2}\right)\right),
$$

where for $r>0$ and $z_{0} \in \mathbb{C}$, we put, as usual, $\Delta\left(z_{0} ; r\right)=\left\{z \in \mathbb{C} ;\left|z-z_{0}\right|<r\right\}$, $\Delta(r)=\Delta(r ; 0)$ and $\Delta=\Delta(1)$. Here $r_{1}, r_{2}, s_{1}, s_{2}$ are positive real numbers such that $r_{2}<r_{1}$ and $s_{2}<s_{1}$. Clearly, the envelope of holomorphy $\widehat{\Omega}_{q}$ is $\Delta^{q}\left(r_{1}\right) \times$ $\Delta^{k}\left(s_{2}\right)$.

A (general) Hartogs figure of order $q$ in $\mathbb{C}^{n}$ is $W_{q}:=F\left(\Omega_{q}\right)$, where $F \in$ $\operatorname{Aut}\left(\mathbb{C}^{n}\right)$. (Notice that this is not the standard definition, as it is given, for instance in [22]!) Clearly, the envelope of holomorphy of $W_{q}$ is $\widehat{W}_{q}$. In this circle of ideas 
we mention Frenkel's lemma (see [1]) stating that $H^{i}\left(W_{q}, \mathcal{O}\right)$ does non-vanishes for $i=0$ or $i=q$. In the same vein one can show that $H^{i}\left(\bar{W}_{q}, \mathcal{O}\right) \neq 0$ if either $i=0$ or $i=q$.

Proof of Proposition 7.1. This follows from [2] with some minor changes. We give only a few points since the reader can easily complete the remaining part of the arguments. For this consider on $\mathbb{C}^{n} \backslash\{0\}$ the form $\eta$ of type $(0, n-1)$ due to Martinelli,

$$
\eta=\left(\left|z_{1}\right|^{2}+\cdots+\left|z_{n}\right|^{2}\right)^{-n} \sum_{j=1}^{n}(-1)^{j-1} \bar{z}_{j} \bigwedge_{1 \leq k \leq n, k \neq j}^{n} \mathrm{~d} \bar{z}_{k}
$$

and $\omega=\mathrm{d} z_{1} \wedge \cdots \wedge \mathrm{d} z_{n}$. For $\alpha=\left(\alpha_{1}, \ldots, \alpha_{n}\right) \in \mathbb{N}^{n}$ and $\xi \in \mathbb{C}^{n}$ we consider a holomorphic mapping $F_{\alpha}^{(\xi)}: \mathbb{C}^{n} \rightarrow \mathbb{C}^{n}$ given by

$$
F_{\alpha}^{(\xi)}(z)=\left(\left(z_{1}-\xi_{1}\right)^{\alpha_{1}}, \ldots,\left(z_{n}-\alpha_{n}\right)^{\alpha_{n}}\right)
$$

Let $K_{\alpha}^{(n)}(\xi)=\omega \wedge\left(F_{\alpha}^{(\xi)}\right)^{\star} \eta$ and $\alpha+1=\left(\alpha_{1}+1, \ldots, \alpha_{n}+1\right)$. Now the version of Proposition 2.3 in [2, page 207] reads:

Proposition 7.2. Let $B$ be the open unit ball in $\mathbb{C}^{n}$. Then for every $\xi \in B$ and any holomorphic function $f$ near $\bar{B}$ one has:

$$
\int_{\partial B} f K_{\alpha+1}^{(n)}(\xi)=\frac{(2 \pi i)^{n}}{(n-1) !} \cdot \frac{1}{\alpha !} \cdot \frac{\partial^{|\alpha|} f}{\partial z^{\alpha}}(\xi) .
$$

To check this, because $\bar{\partial} \eta=0$, the $(n, n-1)$ form $K_{\alpha+1}^{(n)}(\xi)$ is $d$-closed on $\mathbb{C}^{n} \backslash\{\xi\}$ so that if $\Omega$ is a relatively compact open set with smooth boundary in $\mathbb{C}^{n}$ containing $\bar{B}$, then

$$
\int_{\partial \Omega} f K_{\alpha+1}^{(n)}(\xi)=\int_{\partial B} f K_{\alpha+1}^{(n)}(\xi)
$$

and one can take $\Omega$ to be a ball in $\mathbb{C}^{n}$ centered at $\xi$ of radius 2 . By homothety and translation we are reduced to [2] (see there page 207).

Eventually, the infinite dimensionality stated in the "moreover" part follows as in Laufer [24].

Remark 7.3. In studying the existence of Stein neighborhoods of pseudoconvex domains in $\mathbb{C}^{n}$ one encounters a class of examples, the so-called Hartogs domains which are obtained as follows. Start with a Stein open set $\Omega$ in $\mathbb{C}^{n-1}$ and a plurisubharmonic function $\varphi$ on $\Omega$, non-necessarily continuous. Then its Hartogs domain is the set

$$
\Omega_{\varphi}:=\{(z, w) \in \Omega \times \mathbb{C} ;|w|<\exp (-\varphi(z))\} .
$$


Now let $\Omega$ be the open unit ball in $\mathbb{C}^{n-1}$. Select a sequence $\left\{a_{v}\right\}_{v}$ of mutually distinct points that accumulates to every boundary point of $\Omega, a_{v} \neq 0$, and define the plurisubharmonic function $u$ on $\Omega$ by

$$
u(z):=\sum_{v} \epsilon_{v} \log \left\|z-a_{v}\right\|
$$

where the sequence $\left\{\epsilon_{\nu}\right\}_{\nu}$ of positive numbers decreases rapidly to 0 and $u(0)>$ $-\infty$. Then put $D:=\Omega_{\varphi}$, where $\varphi=\exp (u)$. One has readily $(\partial \Omega \times \bar{\Delta}) \cup(\Omega \times$ $\{0\}) \subset \bar{D}$.

Clearly $D$ is a relatively compact Stein domain in $\mathbb{C}^{n}$ and $\bar{D}$ does non-admit a neighbrohood system of $(n-1)$-complete open sets.

Here we follow the $q$-convexity convention as in [1]; namely a real-valued smooth function $\Phi$ of class $C^{\infty}$ defined on an open subset $W$ of $\mathbb{C}^{n}$ is said to be $q$-convex if its Levi form, $L(\Phi, a)$, has at every point $a \in W$ at least $n-q+1$ strictly positive eigenvalues, where

$$
L(\Phi, a)(\xi):=\sum_{\nu=1}^{n} \frac{\partial^{2} \Phi}{\partial z_{i} \partial \bar{z}_{j}}(a) \xi_{i} \bar{\xi}_{j}, \quad \xi \in \mathbb{C}^{n}
$$

We say that $W$ is $q$-complete if $W$ admits a $C^{\infty}$-smooth exhaustion function that is $q$-convex. Both notions extend easily to complex manifolds via holomorphic coordinate charts. A celebrated theorem due to Andreotti and Grauert [1] states that a $q$-complete complex manifold has trivial cohomology in dimension from $q$ on and for coefficients in coherent analytic sheaves.

Therefore, thanks to our proposition, it follows that $H^{n-1}(\bar{D}, \mathcal{O})$ has infinite dimension as a complex vector space.

Note that, if $W \subset \mathbb{C}^{n}$ is $q$-complete and has smooth boundary of class $C^{2}$, then $\bar{W}$ admits a neighborhood system of $(q+1)$-complete open sets, a fortiori $H^{k}(\bar{W}, \mathcal{O})=0$ for $k \geq q+1$. See [40].

\section{References}

[1] A. ANDREOTti and H. GRAUERT, Théorèmes de finitude pour la cohomologie des espaces complexes, Bull. Soc. Math. France 90 (1962), 193-259.

[2] A. Andreotti and F. Norguet, Probléme de Levi et convexité holomorphe pour les classes de cohomologie, Ann. Scuola Norm. Sup. Pisa Cl. Sci. (3) 20 (1966), 187-241.

[3] A. Andreotti and Y.-T. SiU, Projective embedding of pseudoconcave spaces, Ann. Scuola Norm. Sup. Pisa Cl. Sci. (3) 24 (1970), 231-278.

[4] C. BĂNICĂ and O. STĂNĂŞILĂ, Some results on the extension of analytic entities defined out of a compact, Ann. Scuola Norm. Sup. Pisa Cl. Sci. (3) 25 (1971), 347-376.

[5] F.-T. BIRTEL, Some holomorphic function algebras, In: "Summer Gathering on Function Algebras" (Aarhus, 1969), Matematisk Inst., Aarhus Univ., Aarhus, 1969, 11-18.

[6] F.-T. BIRTEL, "Algebras of Holomorphic Functions: 40 Lectures", Tulane University Mathematics Department, New Orleans, 1972. 
[7] J.-E. BJÖRK, Holomorphic convexity and analytic structures in Banach algebras, Ark. Mat. 9 (1971), 39-54.

[8] H. CARTAN, Variétés analytiques réelles et variétés analytiques complexes, Bull. Soc. Math. France 85 (1957), 77-99.

[9] M. COLŢOIU and K. DiEderich, On Levi's problem on complex spaces and envelopes of holomorphy, Math. Ann. 316 (2000), 185-199.

[10] F. DocQuier and H. GRAUERT, Levisches Problem und Rungescher Satz für Teilgebiete Steinscher Mannigfaltigkeiten, Math. Ann. 140 (1960), 94-123.

[11] G. Fischer, "Complex Analytic Geometry", Lecture Notes in Math., Vol. 538, SpringerVerlag, Berlin, New York, 1976.

[12] J.-E. FORNÆSS and R. NARASIMHAN, The Levi problem on complex spaces with singularities, Math. Ann. 248 (1980), 47-72.

[13] M. Furushima, Domains of holomorphy in two dimensional normal Stein spaces, In: "Finite or Infinite Dimensional Complex Analysis and Applications", Kyushu University Press, Fukuoka, 2005, 67-74.

[14] H. Grauert and R. Remmert, Konvexität in der komplexen Analysis. Nich-holomorphkonvexe Holomorphiegebiete und Andwendungen auf die Abbildungstheorie, Comm. Math. Helv. 31 (1956), 152-183.

[15] H. GRAUERT and R. REMMERT, Singularitäten komplexer Mannigfaltigkeiten une Riemannsche Gebiete, Math. Z. 67 (1957), 103-128.

[16] H. GRAUERT and R. REMmERT, "Theorie der Steinschen Räume”, Grundlehren der math. Wiss., Vol. 227, Springer-Verlag, 1977.

[17] R. HARVEY, The theory of hyperfunctions on totally real subsets of a complex manifold with applications to extension problems, Amer. J. Math. 91 (1969), 853-873.

[18] R. HARVEY and R.-O. WELLS, Compact holomorphically convex subsets of a Stein manifold, Trans. Amer. Math. Soc. 136 (1969), 509-516.

[19] A. Hirschowitz, Un example concernant le prolongement analytique, C.R. Acad. Sci. Paris, Sér. A 275 (1972), 1231-1233.

[20] R. IWAHASHI, A characterization of holomorphically complete spaces, Proc. Japan Acad. 36 (1960), 205-206.

[21] R. IWAHASHI, Domains spread on a complex space, J. Math. Soc. Japan 9 (1957), 452-463.

[22] B. KAUP and L. KAUP, "Holomorphic Functions of Several Variables", an introduction to the fundamental theory, with the assistance of Gottfried Barthel, translated from the German by Michael Bridgland, de Gruyter Studies in Mathematics, 3, Berlin, 1983.

[23] H. LAUfER, Some remarks about a theorem of Hartogs, Proc. Amer. Math. Soc. 17 (1966), 1244-1249.

[24] H. LAUfer, On the infinite dimensionality of the Dolbeault cohomology groups, Proc. Amer. Math. Soc. 52 (1975), 293-296.

[25] C. LAURENT-ThiÉBAUt, Sur l'équation de Cauchy-Riemann tangentielle dans une calotte strictement pseudoconvexe, Internat. J. Math. 16 (2005), 1063-1079.

[26] G. LuPACCIOLU, On the envelopes of holomorphy of strictly Levi convex hypersurfqces, In: "Colloque d'Analyse Complexe et Géometrie", Marseille, France 13-17 janvier 1992, Astérisque, Vol. 217 (1993), 183-192.

[27] G. LUPACCIOLU, Characterization of removable sets in strongly pseudoconvex boundaries, Ark. Mat. 32 (1994), 455-473.

[28] A. Martineau, Les hyperfunctions de M. Sato, In: "Séminaire Bourbaki", Vol. 6, Exp. No. 214, Soc. Math. France, Paris, 1995, 127-139.

[29] G. NARdelli and A. TANCREDI, Compatti olomorficamente convessi di uno spazio analitico, Ann. Univ. Ferrara, Sez. VII (N.S.) 25 (1979), 99-108.

[30] P.-J. DE PAEPE, Homomorphism spaces of algebras of holomorphic functions, Pac. J. Math. 66 (1976), 211-220.

[31] H. Rossi, On envelopes of holomorphy, Comm. Pure Appl. Math. 16 (1963), 9-17. 
[32] G. SCHEJA, Fortsetzungssätze der komplex-analytischen Cohomologie und ihre algebraische Charakterisierung, Math. Ann. 157 (1964), 75-94.

[33] H.-W. SCHUSTER, Infinitesimale Erweiterungen komplexer Räume, Comm. Math. Helv. 45 (1970), 265-286.

[34] Y.-T. SiU and G. Trautmann, "Gap Sheaves and Extension of Coherent Analytic Subsheaves", Lect. Notes in Math., Vol. 172, Springer-Verlag, Berlin, 1976.

[35] B. StensoneS, Stein neighborhoods, Math. Z. 195 (1987), 433-436.

[36] O. Stormark, Some properties of compact natural sets in several complex variables, Math. Scand. 33 (1973), 359-374.

[37] T. UedA, Domains of holomorphy in Segre cones, Publ. Res. Inst. Math. Sci. 22 (1986), 561-569.

[38] V. VẦÂATU, Invariance of cohomological q-completeness under finite holomorphic surjections, Manuscr. Math. 82 (1994), 113-124.

[39] V. VÂJÂITU, Holomorphic q-hulls in top degrees, Manuscr. Math. 91 (1996), 195-210.

[40] V. VÂJẦITU, Neighborhoods of Levi q-convex domains, J. Geom. Anal. 8 (1998), 163-177.

[41] W.-R. ZAME, Holomorphic convexity of compact sets in analytic spaces and the structure of algebras of holomorphic germs, Trans. Amer. Math. Soc. 222 (1976), 107-127.

Université des Sciences et Technologies de Lille 1

Laboratoire Paul Painlevé, Bât. M2

F-59655 Villeneuve d'Ascq Cedex, France

Viorel.Vajaitu@math.univ-lille1.fr 